\title{
Short lifetime components in the relaxation of boron acceptors in
} silicon

\author{
K. Saeedi ${ }^{1}$, N. Stavrias ${ }^{1}$, B. Redlich ${ }^{1}$, H. Riemann ${ }^{2}$, N.V. Abrosimov ${ }^{2}$, P. Becker ${ }^{3}$, H-J. Pohl ${ }^{4}$, \\ M.L.W. Thewalt ${ }^{5}$ and B. N. Murdin ${ }^{6}$ \\ ${ }^{1}$ Radboud University, Institute for Molecules and Materials, FELIX Laboratory, Toernooiveld 7c, 6525 ED \\ Nijmegen, The Netherlands \\ ${ }^{2}$ Leibniz-Institut fur Kristallzuchtung (IKZ), 12489 Berlin, Germany \\ ${ }^{3}$ PTB Braunschweig, 38116 Braunschweig, Germany \\ ${ }^{4}$ VITCON Projectconsult, 07743 Jena, Germany \\ ${ }^{5}$ Department of Physics, Simon Fraser University, Burnaby, British Columbia, V5A 1S6, Canada \\ ${ }^{6}$ Advanced Technology Institute and SEPNet, University of Surrey, Guildford, GU2 7XH, United Kingdom
}

\begin{abstract}
We present time-resolved measurements of the relaxation between the orbital states of the shallow acceptor boron in silicon. The silicon host was enriched Si-28, which exhibits life-time broadened absorption lines. We observed a wide range of $T_{1}$ lifetimes from $6 \mathrm{ps}$ to $130 \mathrm{ps}$ depending on the excited state and the pump intensity. The fastest transients have not been observed previously in the time domain, and they are caused by the phonon relaxation responsible for the small-signal frequency domain line-width. We identify the slower components with an ionisation/recombination/cascade pathway.
\end{abstract}

\section{Introduction}

Boron atoms as substitutional impurities in a silicon ( $\mathrm{Si}$ ) host crystal lattice form shallow acceptor centres with a hole binding energy of $45.63 \mathrm{meV}$ [1,2]. Recent proposals utilize the electronic states of the prototypical acceptor boron in silicon as a THz laser medium [3] and as qubits for a silicon based quantum information technology [4]. Both of these applications require detailed knowledge of the orbital-state energies and wavefunctions, and the transition rates between them. Although more attention has been paid to donors, e.g. recently we investigated relaxation in $\mathrm{Si}: \mathrm{Bi}[5]$ the wavefunctions have completely different envelope functions, the valley-orbit terms and the lattice-periodic parts for donors and acceptors, so there is no reason to expect that the matrix elements for phonon relaxation should be similar. For example, the linewidths for Si:Bi monotonically decrease with decreasing binding energy of the excited state [5], which is not true for Si:B. Varieties of electric-dipole-allowed transitions for $\mathrm{Si}: \mathrm{B}$ are readily observed by infrared (IR) transmission spectroscopy, and have received detailed experimental and theoretical study in the past [2]. States with odd parity are sometimes labelled with a superscript - which we drop here for simplicity since all the excited states are odd (and the even parity ground state would have a superscript + ). The spectral widths of the Lyman-series transitions (i.e. starting from the ground state) for isotopically enriched ${ }^{28} \mathrm{Si}$ host exhibit a wide variation depending on the excited state; absorption lines for the ground state to some of the lower energy excited states (e.g. transitions from ground state to $1 \Gamma_{8}, 2 \Gamma_{8}$ ) have considerably wider full width at half maximum (FWHM) than those than for higher lying states (e.g. $\left.3 \Gamma_{7}\right)[6]$.

For a homogeneously broadened Lorentzian line, the dephasing time is $T_{2}=2 / \Delta \omega=1 / \pi \Delta v$ where $(\Delta \omega) \Delta v$ is the FWHM in (angular) frequency. For an inhomogeneously broadened line $\Delta v$ $>1 / \pi \mathrm{T}_{2}$ and the line shape is (usually) Gaussian. The high-lying absorption lines are susceptible to inhomogeneous concentration broadening, but if care is taken to produce single crystals with high chemical and isotopic purity, and low strain, the low-lying lines appear Lorentzian [6], so 
the equality seems established in this case. If the only dephasing process is the population loss from the excited state, $T_{2}=2 T_{1}$ but if there is extra dephasing in addition to the population decay then $T_{2}<2 T_{1}$. In short, the linewidth $\Delta v \geq 1 / \pi T_{2} \geq 1 / 2 \pi T_{1}$, and the purpose of the present study is to establish whether $T_{1}=1 / 2 \pi \Delta v$, in which case we have no sources of inhomogeneity or extra dephasing, i.e. lifetime-broadened lines.

The orbital dynamics of boron doped ${ }^{\text {nat }} \mathrm{Si}$ has previously been studied using time-domain spectroscopy with the FELIX free-electron laser [7], and the transients exhibited several dynamical time-scales much longer than $1 / 2 \pi \Delta v$. The spectral widths [6] were larger than the inverse decay times [7] by a factor of between 2 to 8 depending on the line. The mechanisms responsible for the long decay times were unclear, as was the absence of the correspondingly sharper components in the small-signal spectra. In this work we show that careful control of the intensity, temperature and pulse duration in the time-domain experiments brings the frequency and time-domain measurements into accord.

Experimental setup and procedure

61 The samples used in this study were cut from a ${ }^{28} \mathrm{Si}$ crystal doped with a boron concentration 62 of $[B]=4 \times 10^{14} \mathrm{~cm}^{-3}$, a low phosphorus concentration of $[\mathrm{P}]=5 \times 10^{11} \mathrm{~cm}^{-3}$ and negligible other electrically active impurities. This material has the same enrichment as the sample that was used for the earlier high resolution spectroscopy [6]. For comparison the sample used in the earlier time domain work [7] was Float Zone grown silicon with natural isotopic composition and similar doping level $\left(\sim 10^{14} \mathrm{~cm}^{-3}\right)$. The new material has higher boron doping concentration than earlier work [6], which increases the absorbance and results in more concentration broadening of the higher energy lines. Here we only investigated (low-lying) lines.

Unless otherwise mentioned all experiments were performed in a Janis super-vari-temp (SVT200) cryostat of the same type as used for earlier spectroscopy [6] with sample mounted in either flowing helium vapour or immersed in superfluid helium, with HDPE windows on the outer vacuum chamber and cold polypropylene windows on the sample chamber was used. The sample was mounted strain free on a PCB using two copper strips. The sample temperature was monitored using a calibrated diode temperature sensor. For some experiments we used an Oxford Instruments microstat of the same type used as previous pump-probe measurements [7] with room temperature polypropylene windows. The sample was mounted in vacuum on a cold finger, and the temperature was monitored with a rhodium-iron sensor on the heat-exchanger above the sample finger. In what follows we refer to the latter cryostat as "sample in vacuum".

One sample was polished to a wedge, and then chemically etched to remove any surface damage which would otherwise strain the sample and consequently could split and/or broaden the optical transitions. The high resolution Fourier Transform Infrared (FTIR) absorbance spectrum of this sample is shown in Fig 1. The position and peak height of the experimental absorption lines match very well with expectation. The expected centre frequencies on Fig 1 were taken from Ref [6] and the relative heights were found from the ratio of the oscillator strength (OS), $f$, and the line width, $\Delta v$ (appropriate for well resolved absorption lines) from Refs [8] and [6] respectively. The experimental FWHM was the same for both this and the older sample [6]. Note that the boron has natural isotopic composition (80:20) and the dominant contribution is from ${ }^{11} \mathrm{~B}$ but some ${ }^{10} \mathrm{~B}$ components are visible, shifted $4.6 \mathrm{GHz}$ higher, in the case of the sharpest lines $1 \Gamma_{6}$ and $3 \Gamma_{7}$. Some Si:P lines are also visible e.g. the $1 \mathrm{~s}-2 \mathrm{p}_{0}$ at 8.24699

90 THz. 
A second sample from the same crystal was produced for the pump-probe spectroscopy. It was a $0.44 \mathrm{~mm}$ thick wafer, cut to $1 \times 1 \mathrm{~cm}$ (without a wedge) and then chemically etched as for the other sample. The optical arrangement for the pump-probe experiment with a balanced arm was the same as for earlier work [7] and for more detail on the experimental setup see [5]. FELIX provided the intense, picosecond pulses in the terahertz region of interest required to perform time-resolved, energy-relaxation experiments in a pump-probe geometry.

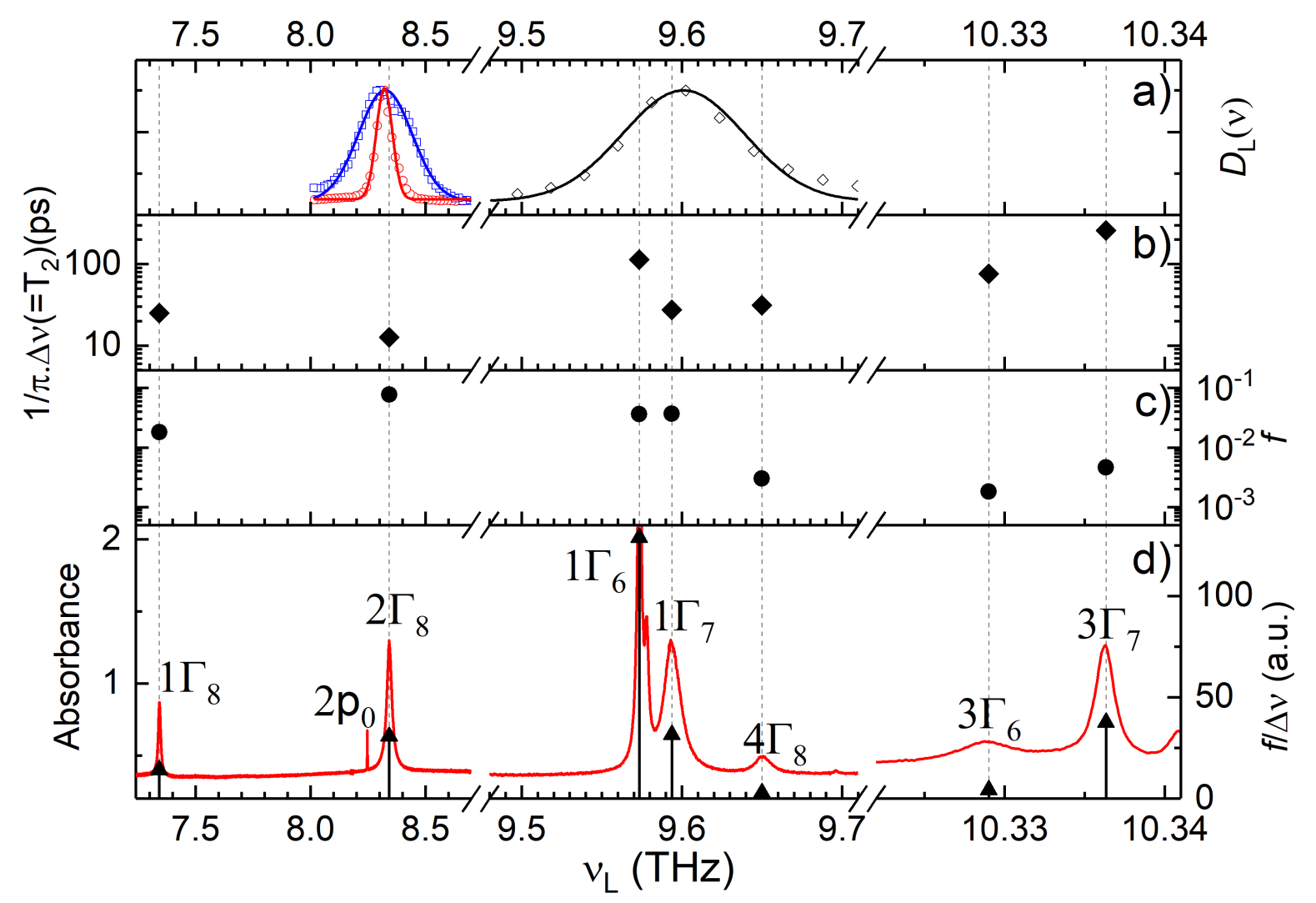

Fig 1. The absorption spectrum of ${ }^{28} \mathrm{Si}: \mathrm{B}$ and the FELIX laser spectrum. (a) The laser spectral density, $D_{\mathrm{L}}(v)$, for some different settings of the cavity length detuning used: $v_{\mathrm{L}}=8.33 \mathrm{THz} \& \Delta v_{\mathrm{L}}=98 \mathrm{GHz}\left(\Delta t_{\mathrm{L}}=4.5 \mathrm{ps}\right)$, red; $v_{\mathrm{L}}=8.33 \mathrm{THz} \& \Delta v_{\mathrm{L}}=255 \mathrm{GHz}\left(\Delta t_{\mathrm{L}}=1.7 \mathrm{ps}\right)$, blue; $v_{\mathrm{L}}=9.60 \mathrm{THz} \& \Delta v_{\mathrm{L}}=90 \mathrm{GHz}\left(\Delta t_{\mathrm{L}}=4.9 \mathrm{ps}\right)$, black. (b) The inverse linewidth, $1 / \pi \Delta v(\log$ scale), where $\Delta v$ is the FWHM linewidth in frequency from Ref [6] (instrument resolution $0.2 \mathrm{GHz}$ ), which is equal to $T_{2}$ in the case of homogeneously broadened lines. (c) $T$ The oscillator strengths, $f$, (log scale) from Ref [8], which determine the excitation and detection in our short pulsed laser experiment. (d) The absorbance spectrum of ${ }^{28} \mathrm{Si}: \mathrm{B}$, produced with high resolution FTIR spectroscopy. Also shown is the product of the OS from (c) with inverse width (b), which is proportional to the peak cross-section for well resolved transitions. Transitions are labelled according to the excited state Refs [5,7]. A Si:P line is visible, labelled $2 \mathbf{p}_{0}$.

\footnotetext{
A unique feature of synchronously pumped lasers like FELIX is that the pulse duration can be controlled by detuning the cavity round-trip frequency away from the pump repetition frequency. The laser pulse's spectral energy density $D_{\mathrm{L}}(v)$ is approximately Gaussian, as shown in Fig 1a. The laser spectra were produced using a grating spectrometer with a linear array of pyroelectric detectors, and in this work, the mean and r.m.s. width in wavelength, $\lambda \&$
} 

using $\Delta v_{\mathrm{L}}=\left(\sigma_{\lambda} / \lambda\right) \cdot(\mathrm{c} / \lambda) \cdot 2 \sqrt{ }(2 \ln 2)=706 \mathrm{THz} \cdot \mu \mathrm{m}\left(\sigma_{\lambda} / \lambda\right) / \lambda$ appropriate for a Gaussian spectrum

118 with small $\sigma_{\lambda}$. The FELIX pulses are approximately transform limited [9] in which case the electric field is well approximated by a pure sinusoid with a Gaussian envelope, from which it can be shown that the FWHM of the intensity profile in time is $\Delta t_{\mathrm{L}}=2 \ln 2 / \pi \Delta v_{\mathrm{L}}=0.441 / \Delta v_{\mathrm{L}}$ (or alternatively $\Delta t_{\mathrm{L}}$ is half of the FWHM duration in time of the Fourier Transform of the intensity spectrum in frequency). As mentioned above, $T_{1} \geq T_{2} / 2 \geq 1 / 2 \pi \Delta v$ and the shortest possible timescale for $\mathrm{T}_{1}$ occurs when $\mathrm{T}_{1}=\mathrm{T}_{2} / 2=1 / 2 \pi \Delta \mathrm{v}$. We therefore require that the laser pulses are significantly shorter than this, i.e. $\Delta t_{\mathrm{L}}<<\mathrm{T}_{1}$ so $\Delta \mathrm{v}_{\mathrm{L}}>00.883 / \mathrm{T}_{2}$. Values for $\mathrm{T}_{2}$ are shown in Fig $1 b$.

\section{Results and Analysis}

127 Pump probe results for a variety of transition lines, laser intensities, laser pulse widths and temperatures are shown in Figs 2, 3, and 4.

So long as the pulse duration is short compared to the relaxation and the relaxation is exponential, then the pump-probe signal may be approximated by

$S(t)=C+\frac{1}{2}\left[1+\operatorname{erf}\left(\frac{t-t_{0}}{t_{\mathrm{R}}}\right)\right] \sum_{i} A_{i} \exp \left(-\frac{t-t_{0}}{T_{1 i}}\right)$

where the factor involving the error function with rise time $t_{\mathrm{R}}$ represents the smooth population increase proportional to the integral of the pump pulse profile, convolved with the probe pulse. Although this model is for weak, incoherent excitation, we find that it produces a good functional agreement with the data in this strong coherent experiment. Integrating a Gaussian pump pulse with linear excitation and convolving with a Gaussian probe pulse of the same width, it can be shown that $t_{\mathrm{R}}=\Delta t_{\mathrm{L}} / \sqrt{ }(2 \ln 2)=\Delta t_{\mathrm{L}} / 1.177$ (where $\Delta t_{\mathrm{L}}$ is the FWHM of the laser pulse duration as mentioned before). At large negative delay time the balancing technique should insure that $S$ is close to zero and $C$ should therefore be close to 0 , just as we see experimentally. Non-zero $C$ is simply an artefact of imperfect balancing. The arrival time of the pump, $t_{0}$, is arbitrary but fixed to be the same for all experiments. Each exponential decay term in Eqn (1) has amplitude, $A_{i}$, and lifetime, $T_{1 i}$.

In the case that only one transition is excited, and the decay is a simple relaxation from the excited state to the ground state we expect that there is only one single exponential decay term in Eqn (1). For short pulses the laser spectrum is broad (Fig 1a) and can overlap with multiple lines, producing multiple decay components. For example Fig 1a,b shows that with the laser centred at $9.60 \mathrm{THz}$ we would expect the transient to be dominated by $1 \Gamma_{7}$, but with a contribution from $1 \Gamma_{6}$ (with $A$ approximately four times smaller) and so there should be two components in the decay.

Relaxation out of the excited state back to the ground state might proceed via an intermediate state. If such an intermediate state is long lived, it would give rise to double exponential decay with equal amplitude components $\left(A_{e \rightarrow i}=A_{i \rightarrow g}\right)$, independent of intensity. This is because the probe absorption is proportional to $\mathrm{n}_{\mathrm{g}}-\mathrm{n}_{\mathrm{e}}$, which is equally sensitive to both the depletion of the excited state (population $\mathrm{n}_{\mathrm{e}}$ ) and the re-filling of the ground state (population $\mathrm{n}_{\mathrm{g}}$ ). If the intermediate state is metastable (short-lived) then it has no effect on the observed transient absorption, i.e. a pump-probe experiment cannot distinguish between direct relaxation and relaxation via meta-stable states. At elevated temperature or high intensity, there is also a possibility of ionisation and recombination. This manifests itself as a reciprocal decay if the 
recombination is the slowest step in the process, and exhibits a strong intensity dependence. However, if the recombination involves highly excited states and the subsequent cascade relaxation back to the ground state is slow, then this pathway can produce an exponential decay. A slow exponential ionization/recombination/cascade mechanism is distinguishable from relaxation via long-lived intermediate states because the former are intensity dependent and disappear at low power while the latter have fixed equal amplitude. The former is obviously present in the intensity dependences of Figs $2 \mathrm{a}\left(2 \Gamma_{8}\right)$ and $4 \mathrm{a}\left(1 \Gamma_{6}\right.$ and $\left.1 \Gamma_{7}\right)$, and there is no evidence of long-lived intermediate states in these cases. The transitions to $1 \Gamma_{8}$ (Fig $3 a$ ) and $3 \Gamma_{7}$ (Fig 3b) have smaller OS (Fig 1c) and further reduction of the laser intensity was inconclusive.

We fitted Eqn (1) to the experimental transients (Figs 2, 3, and 4) with the number of exponential components fixed by the number of lines overlapped by the laser (Fig 1). Unless otherwise specified (the case of Fig $2 \mathrm{~b}$ ), in all cases we fixed $\mathrm{T}_{1 i}$ to the appropriate value(s) of $\mathrm{T}_{2 i} / 2$ from the frequency domain data, i.e. we did not allow $\mathrm{T}_{1 i}$ to be a free parameter. The free parameters were $t_{0}, t_{\mathrm{R}}, C$ and $A_{i}$. Where the resulting residuals showed an obvious time dependence we then added a constant term to the decay (i.e. a very long time-scale decay) corresponding to the ionisation/recombination/cascade, and where the resulting residuals still showed an obvious time dependence, we replaced the constant term with an exponential with time constant $\tau$ being a free parameter. The final residuals are shown in each figure. With this procedure, we assume that the frequency-domain lines are not only homogeneously broadened

182 $\left(\mathrm{T}_{2}=1 / \pi \Delta v\right)$ due to the isotopically enriched silicon host, but they are also lifetime-broadened $\left(\mathrm{T}_{1}=\mathrm{T}_{2} / 2\right)$. Allowing for the possibility of one additional pathway, we test for convincing evidence that this is false (and as we shall see, we find none).
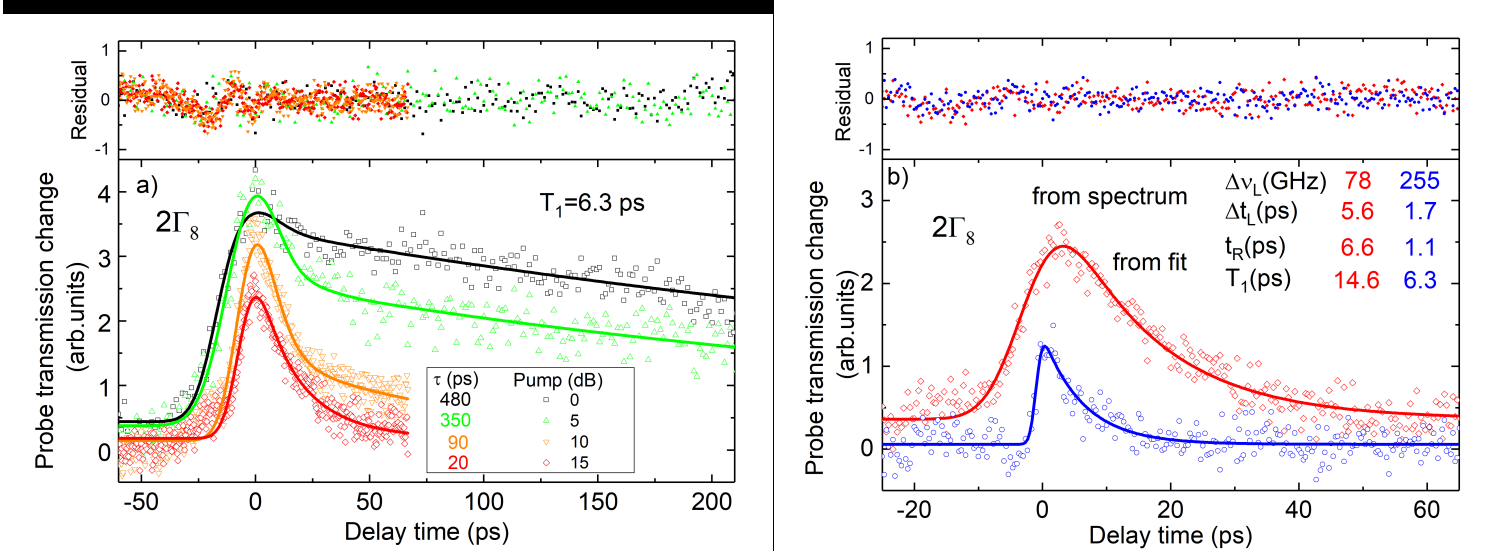

Fig 2. The change in transmission of the probe pulse as a function of the delay between pump and probe, for a laser centre frequency of $8.33 \mathrm{THz}$ for excitation to $2 \Gamma_{8}$. a) The dependence on laser attenuation in $\mathrm{dB}$. The sample was at a temperature of approximately $6 \mathrm{~K}$. The spectral width was $\Delta v_{L}=78 \mathrm{GHz}$ (i.e. $\Delta t_{\mathrm{L}}=5.6 \mathrm{ps}$ ). Fits had two components, a fixed $T_{1}=T_{2} / 2(6.3 p s)$ and a longer component with lifetime $\tau$ that was a free parameter. b) Laser pulse duration dependence at $T=6 \mathrm{~K}$ and a laser attenuation of $15 \mathrm{~dB}$ (where $0 \mathrm{~dB}$ corresponds to a micropulse energy of $180-300 \mu \mathrm{J}$ in front of the cryostat; we estimate roughly $30 \%$ loss on the way into the sample, with a $1-2 \mathrm{~mm}$ focus on the sample position). The laser spectral width $\Delta v_{\mathrm{L}}$ (and corresponding $\Delta t_{\mathrm{L}}$ ) is shown on the figure, along with the rise time, $t_{R}$, from the fit. Blue: short pulses and $T_{1}$ fixed at 6.3ps; Red: longer pulses with $T_{1}$ a free parameter. The red data is the same in both $a \& b$. 

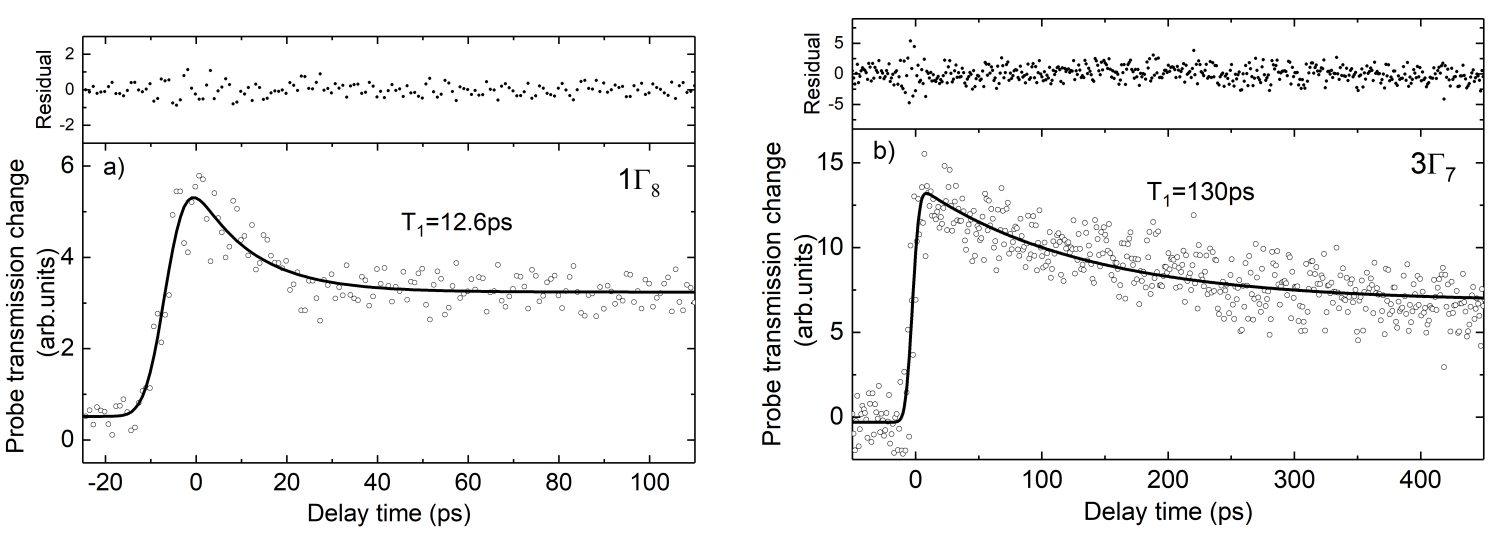

Fig 3. The change in transmission of the probe pulse as a function of the delay between pump and probe a) for a laser centre frequency of $7.35 \mathrm{THz}$ for excitation to $1 \Gamma_{8}$. The laser spectral width was $\Delta v_{\mathrm{L}}=69 \mathrm{GHz}$ (i.e. $\Delta t_{\mathrm{L}}=6.4 \mathrm{ps}$ ). The pump attenuation was $0 \mathrm{~dB}$. The fit line had fixed decay of $T_{1}=T_{2} / 2(=12.6 \mathrm{ps})$ and a constant component. b) For a laser centre frequency of $10.338 \mathrm{THz}$ with a width of $\Delta v_{\mathrm{L}}=97 \mathrm{GHz}$ (i.e. $\Delta t_{\mathrm{L}}=4.6 \mathrm{ps}$ ). The laser produces excitation mainly to $3 \Gamma_{7}$ and is only weakly overlapping with $3 \Gamma_{6}$. The pump attenuation was 10dB. The fit line has fixed decay of $T_{1}=T_{2} / 2(=130 \mathrm{ps})$ and a constant component. For both cases sample was at $T=8 \mathrm{~K}$ and the rise time from the fit was $t_{\mathrm{R}}=6 \mathrm{ps}$.
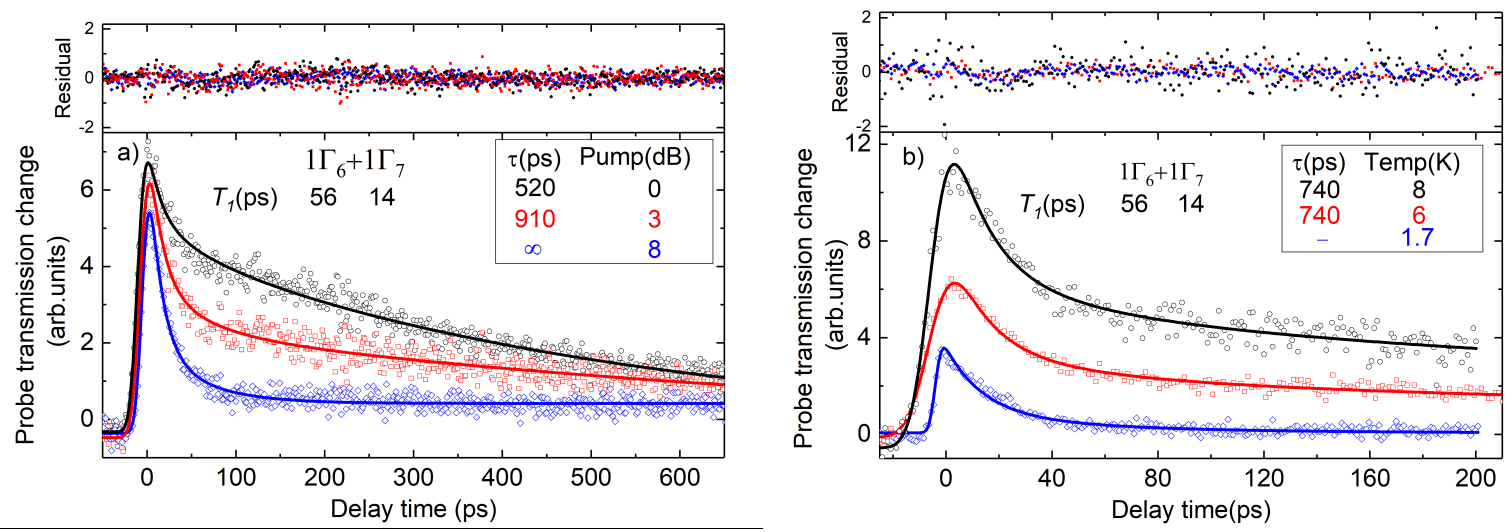

Fig 4. The change in transmission of the probe pulse as a function of the delay between pump and probe, for a laser centre frequency of $9.60 \mathrm{THz}$ for excitation to $1 \Gamma_{7}$ and $1 \Gamma_{6}$. The fits included 2 decay components with variable amplitude and fixed decay, $T_{1}=T_{2} / 2$ $\left(T_{1 \mathrm{a}}=14\right.$ and $\left.T_{1 \mathrm{~b}}=56 \mathrm{ps}\right)$, and a long variable lifetime component. a) Intensity dependence for $T=6 \mathrm{~K}$, for a laser spectral width $\Delta v_{\mathrm{L}}=90 \mathrm{GHz}\left(\Delta t_{\mathrm{L}}=4.9 \mathrm{ps}\right)$. For a pump attenuation of OdB the micropulse energy was $300 \mu \mathrm{J}$ in front of the cryostat (we estimate roughly $30 \%$ loss on the way into the sample, with a $1-2 \mathrm{~mm}$ focus on the sample position). The fitted rise time was $t_{R}=9 \mathrm{ps}$. The fitted amplitudes for the fixed lifetime components were in the ratio 4:1 with the faster decay being stronger, consistent with the stronger overlap with $1 \Gamma_{7}$. The lifetime of the longest component is given on the legend. $\left.b\right)$ The temperature dependence at a pump micropulse pulse energy of $35 \mu \mathrm{J}$. Note that the laser spectral width was increased for the lowest temperature to improve temporal resolution. The fitting was as for (a), and the lifetimes for the longest component are shown on the legend. At the 
Fig 2a shows the intensity dependence of the pump-probe transient for excitation to $2 \Gamma_{8}$. The decay lifetimes of the results match the previous work [7], but they are significantly longer than the $\mathrm{T}_{1}=6.3 \mathrm{ps}$ expected from the linewidth. The decay has two components, and the strength of the long component rises as the laser intensity is increased. This can be explained by an increase in the population of the excited state with intensity, and a consequent increase in photo-ionisation. We might expect that the amplitude of the component associated with photo-ionisation would be proportional to intensity squared, but the aim of this work was to investigate correspondence of the lifetime at low intensity with small-signal line-width and we did not investigate this in detail. The associated lifetime also rises and appears to saturate as the long component becomes dominant. At the highest intensities the longer component of decay seems exponential. Since the recombination of free-holes with the negatively charged acceptor ions must exhibit a reciprocal decay, the recombination step cannot be the slowest part of the return process for the ionised holes. Clearly, once the recombination has taken place the holes are in highly excited bound states, and the cascade relaxation back to the ground state takes significantly longer. This is consistent with the very sharp lines exhibited by highly excited state in the small signal absorption [6].

Fig $2 \mathrm{~b}$ shows the pump-probe transient for excitation to $2 \Gamma_{8}$ at low intensity for different laser pulse durations. This absorption line is well separated from any others and we expect a single exponential decay at low intensity. In experiments where the laser spectrum is very broad (and hence the pulse short), this is indeed what we find - the fit with a fixed $\mathrm{T}_{1}=\mathrm{T}_{2} / 2(=6.3 \mathrm{ps}$ from Fig 1) agrees very well with the data with no additional components. For $\Delta v_{\mathrm{L}}=255 \mathrm{GHz}$ the inferred $\Delta t_{\mathrm{L}}=1.7 \mathrm{ps}$, and we would therefore expect $t_{\mathrm{R}}=1.4 \mathrm{ps}$ for a weak, incoherent excitation, in very good agreement with the fit value $(1.1 \mathrm{ps})$ in spite of this being a strong, coherent experiment. Reducing the laser spectral width by a third triples the laser pulse duration and clearly also the rise time of the transient, but this also has a significant effect on the observed decay, in part due to the simple convolution effect of the probe. The fit shown (red curve) used Eqn (1) with a single exponential decay with apparent lifetime $T_{1}=14.6 \mathrm{ps}$ as a free parameter (unlike the fit to the same data in Fig $2 \mathrm{a}$ where we fixed $\mathrm{T}_{1}$ to the inverse line-width and added a second component). This value is clearly much larger than the lifetime from the short pulse data (blue curve). It seems likely that the increase in recovery time with reducing spectral width also comes in part due to better coupling of the laser to the absorption line and therefore increased excitation: during a coherent pumping the wavefunction amplitude rises with $\Omega_{\mathrm{R}} \Delta t_{\mathrm{L}}$ where $\Omega_{\mathrm{R}}$ is the Rabi frequency, i.e. the excitation probability rises with $\Omega_{\mathrm{R}}^{2} \Delta t_{\mathrm{L}}^{2} \propto f I_{\mathrm{L}} \Delta t_{\mathrm{L}}^{2} \propto$ $f E_{\mathrm{L}} \Delta t_{\mathrm{L}}$ where $I_{L}$ and $E_{L}$ are the laser pulse intensity and energy respectively. Therefore the longer the pulse the greater the excitation, and photo-ionisation was seen when we increased $I_{L}$ (and $E_{L}$ ) which directly increases the excitation, as shown in Fig 2a.

In the case of excitation to $1 \Gamma_{8}$, the pump probe transient shown in Fig $3 \mathrm{a}$ has a clear fast transient and a long lived additional component. The fit is quite consistent with $T_{1}=T_{2} / 2$ (=12.6ps) for the fast component lifetime.

Setting the laser to $10.338 \mathrm{THz}$ (Fig $3 \mathrm{~b}$ ) produces excitation primarily to $3 \Gamma_{7}$, which has a very long $\mathrm{T}_{2 \mathrm{a}}$ of $260 \mathrm{ps}$, but also to $3 \Gamma_{6}$ which has a significantly smaller OS and also a significantly smaller $T_{2 b}$ of 75 ps (Fig 1b). In the fit to the pump-probe transient (Fig 3b) we found that the data are consistent with faster component having negligible contribution and only the $\mathrm{T}_{1 \mathrm{a}}=\mathrm{T}_{2 \mathrm{a}} / 2$ (=130ps) component being important (with an additional constant component).

263 The situation for the data of Fig 4 is more complicated, because there are two components of similar OS $1 \Gamma_{6}$ and $1 \Gamma_{7}$ (and a third component $4 \Gamma_{8}$ which has negligible OS compared with 
the other two). The $1 \Gamma_{7}$ line dominates the small-signal high-resolution transmission spectrum

266 (Fig 1d) because it is far sharper, even though the other $\left(1 \Gamma_{6}\right)$ has slightly larger OS, Fig 1c. As already mentioned, with the laser centred on $9.60 \mathrm{THz}$ the overlap with $1 \Gamma_{7}$ is somewhat better. The relative contributions from each transition to the pump probe experiment may be found from the OS, $f$, and the laser spectral density at the transition frequency, $D_{L}(v)$, Fig 1a. The function is complicated in the case of a highly coherent quantum oscillator and a coherent pulse, but for a weak, incoherent experiment the excited population due to the pump is $\Delta n \propto$ $f D_{\text {pump }}(v) \Delta v$, and the absorption change of the probe is $\Delta \alpha \propto f \Delta n$. Since the pulse energy density at the transition frequency for the pump and probe $D_{\text {probe }}(v) \propto D_{\text {pump }}(v) \propto D_{\mathrm{L}}(v)$, then $S(v) \propto \Delta \alpha D_{\text {probe }}(v) \Delta v \propto D_{L}^{2}(v) f^{2}$. Since we did not spectrally resolve the pump-probe experiment we simply see the sum of the decays at each frequency. The fit to the data of Fig 4 show that the components have amplitude $A_{i}$ in the ratio $4: 1$ at the lowest temperature and lowest intensity, and from the approximation from an incoherent experiment just given this would imply $D_{\mathrm{L}}(v) f \sim 2$, which is reasonably consistent with the data in Fig 1a,c considering this is a coherent experiment. This situation does not change very much as the temperature or intensity is increased, but it is evident that there is a significant effect on the long-time constant component, which decreases in lifetime and increases in strength. We also performed the experiment of Fig $4 \mathrm{~b}$ with the sample in vacuum, for comparison (data not shown). The same qualitative behaviour was seen, with very slightly longer lifetimes as might be expected if the sample temperature was elevated with respect to the sensor reading.

As already mentioned, in previous pump-probe work on Si:B [7] the $\mathrm{T}_{1}$ times (which were obtained by unrestricted fits) were considerably longer than those used in Figs 2-4. In the case of Fig $4\left(1 \Gamma_{6}\right.$ and $\left.1 \Gamma_{7}\right)$, the results are a combination of components with $T_{2} / 2=14 \mathrm{ps}$ and $56 \mathrm{ps}$, with equal OS and potentially equal contribution - the previous work gave $25 \mathrm{ps}$ for the lifetime and it seems plausible that this was simply averaged. On the other hand, we have shown here that much shorter lifetimes can be obtained for Fig $3 \mathrm{a}$ and $2 \mathrm{~b}\left(1 \Gamma_{8}\right.$ and $2 \Gamma_{8}$ with $\mathrm{T}_{2} / 2=12.6$ and $6.3 \mathrm{ps}$ respectively) than previously assigned $\left(\mathrm{T}_{1}=55 \mathrm{ps}\right.$ and $45 \mathrm{ps}$ respectively) [7]. The fast dynamics has been resolved here by ensuring that the pulse duration was reduced carefully, and that the temperature and intensity were as low as possible. The previous longer lifetimes were clearly partly limited by the pulse duration: we showed in Fig $2 b$ that it is not sufficient to ensure that the rise time is shorter than the apparent decay, because the coupling of the laser to the excitation is a function of pulse duration. The temperature also seems to be an issue Fig $4 \mathrm{~b}$ shows that raising the temperature has a significant effect on the ionization/recombination/cascade process that becomes mixed in with the direct relaxation. High intensity can be responsible for increased photo-ionisation, and therefore new relaxation pathways, as observed in Fig 2a and 4a. Finally, we note that Ref [7] includes a photon echo experiment for the $1 \Gamma_{8}$ line that produces a decay of $T_{2} / 4=17.25 \mathrm{ps}$ and hence an inferred $\mathrm{T}_{2}=69 \mathrm{ps}$. The present results are consistent with $\mathrm{T}_{2}=25 \mathrm{ps}$, which would indicate that the photon echo decay should have been $T_{2} / 4=6 \mathrm{ps}$, i.e. less than the pulse duration used ( $\left.7 \mathrm{ps}\right)$ and not resolvable. Even with the shortest pulses used here (1.5ps) a $6 \mathrm{ps}$ echo decay would be challenging to observe so it seems likely that the echo was simply resolution limited.

\section{Summary}

307 We have found that the linewidth, $\Delta v$, measured from high resolution spectroscopy is perfectly consistent with pump probe data for the absorption lines studied here. In the case of transitions from ground state to $1 \Gamma_{8}, 2 \Gamma_{8}, 1 \Gamma_{7}, 1 \Gamma_{6}$, and $3 \Gamma_{7}$ we find that the transient decay (with the 310 shortest pulses at the lowest temperature and lowest intensity) is well described by $\mathrm{T}_{1}=1 / 2 \pi \Delta \mathrm{v}$,
311 consistent with lifetime broadened lines with no extra dephasing $\left(\mathrm{T}_{1}=\mathrm{T}_{2} / 2\right)$ and no 
312 inhomogeneous broadening $\left(\mathrm{T}_{2}=1 / \pi \Delta v\right)$. There was a long time-constant component (often

313 with decay time much longer than the length of our delay line, i.e. with lifetime $\tau>>1 \mathrm{~ns}$ ) in

314 almost all the fits. Where the OS was large enough to get data over a range of intensities $\left(1 \Gamma_{6}\right.$,

$\left.3151 \Gamma_{7}, 2 \Gamma_{8}\right)$ the long lived components were seen to quench in strength with reducing intensity,

316 and this correlated with an increase in time constant, so it seems more likely that they result

317 from an ionization/recombination/cascade process rather than a long-lived intermediate state.

318 Acknowledgements

319 We gratefully acknowledge the Nederlandse Organisatie voor Wetenschappelijk 320 Onderzoek (NWO) for the support of the FELIX Laboratory and the financial support from the 321 UK Engineering and Physical Sciences Research Council [COMPASSS/ADDRFSS, Grant No. 322 EP/M009564/1]. B.N.M. is grateful for a Royal Society Wolfson Research Merit Award.

\section{References}

324 [1] E. Burstein, E. E. Bell, J. W. Davisson, M. Lax, J. Phys. Chem. 57, 849 (1953).

325 [2] B. Pajot, Springer Series in Solid-State Sciences, Vol. 158 (2010).

326 [3] S. G. Pavlov, et al. Phys. Rev. X 4, 021009 (2014)

327 [4] J. Salfi, J. A. Mol, D. Culcer, S. Rogge, Phys. Rev. Lett. 116, 246801 (2016)

328 [5] N. Stavrias, et al. Phys. Rev. B 96, 155204 (2017)

329 [6] M. Steger, et al. Phys. Rev. B 79, 205210 (2009)

330 [7] N. Q. Vinh, et al. Phys. Rev. X 3, 011019 (2013)

331 [8] R.A. Lewis, P. Fisher, N.A. McLean, Aust. J. Phys. 47, 329-360 (1994)

332 [9] G. M. H. Knippels, R. F. X. A. M. Mols, A. F. G. van der Meer, D. Oepts, P. W. van

333 Amersfoort, Phys. Rev. Lett. 75, 1755 (1995) 